\title{
Unexpected Behavior of the Stopping of Slow Ions in Ionic Crystals
}

\author{
J. I. Juaristi, ${ }^{1}$ C. Auth, ${ }^{2}$ H. Winter, ${ }^{2}$ A. Arnau, ${ }^{1}$ K. Eder, ${ }^{3}$ D. Semrad,${ }^{3}$ F. Aumayr, ${ }^{4}$ P. Bauer, ${ }^{3, *}$ and P. M. Echenique ${ }^{1}$ \\ ${ }^{1}$ Departamento de Física de Materiales, Facultad de Químicas, Apartado 1072, E-20080 San Sebastián, \\ Spain and Centro Mixto C.S.I.C-UPV/EHU \\ ${ }^{2}$ Institut für Physik, Humboldt Universität zu Berlin, Invalidenstrasse 110, D-10115 Berlin, Germany \\ ${ }^{3}$ Institut für Experimentalphysik, Johannes-Kepler-Universität, Altenberger Strasse 69, A-4040 Linz, Austria \\ ${ }^{4}$ Institut für Allgemeine Physik, Technische Universität Wien, Wiedner Haupstrasse 8-10, A-1040 Wien, Austria
}

(Received 8 November 1999)

\begin{abstract}
The energy loss of slow ions during grazing scattering from a $\operatorname{LiF}(100)$ surface as a function of the projectile atomic number $Z_{1}$ is observed to show oscillations similar to those occurring in metals. A model of stopping of ions in an electron gas where screening is calculated from density functional theory reproduces well the experimental data. The same model gives good agreement with the energy loss obtained in transmission experiments performed with $\mathrm{H}$ and $\mathrm{He}$ projectiles. Analysis of these results allows us to gain new insights in the stopping of slow ions in ionic crystals.
\end{abstract}

PACS numbers: $34.50 . \mathrm{Bw}, 78.90 .+\mathrm{t}, 79.20 . \mathrm{Rf}$

Recently, experiments were performed on the energy loss of low velocity protons interacting with wide band gap insulators [1-3] which led to new aspects on electronic stopping of ions in solids. For metals a linear velocity dependence of the energy loss is found. The predominant role played by excitations of conduction band electrons close to the Fermi level is the reason for this [4]. However, for insulators, as in gaseous targets [5], these low energy excitations are expected to be suppressed due to an energy gap. This would imply a threshold effect for projectile stopping with respect to ion velocity [6,7] and therefore a deviation from the linear velocity dependence of the stopping power. In transmission experiments with a LiF target no threshold effect was observed down to $2.5 \mathrm{keV}$ proton energies [2] that correspond to a velocity $v \approx 0.32$ atomic units (a.u.) [8]. This finding was interpreted as a local reduction of the electronic band gap in close collisions of the projectiles with the target atoms, enabling low energy electronic excitations. In this respect, it is important to state that electron promotion via molecular orbital formation also explains the measured spectra of electrons emitted in slow proton-LiF surface collisions [9]. In surface scattering experiments a threshold effect was observed below $2 \mathrm{keV}$ [3], energies not accessible so far in transmission experiments. From an analysis of the energy loss spectra, electronic charge exchange processes were identified as the dominant mechanism of electronic stopping of protons in $\mathrm{LiF}$ [3]. In this regime, combining in coincidence energy loss and electron emission, Roncin et al. [10] identified surface exciton population as an energy loss channel.

In a new combined theoretical and experimental study we explore and explain in a consistent manner the energy loss processes in the interaction of slow ions with insulators and resolve the seeming discrepancy between the transmission and surface scattering experiments. We have performed energy loss measurements for surface scattering as well as for thin foil transmission. We present re- sults obtained for the energy loss of $v=0.5$ a.u. ions with atomic number $Z_{1}=1-20$ scattered off a $\operatorname{LiF}(100)$ surface under grazing angle of incidence $\Phi_{\text {in }}=1^{\circ}$, and measurements of the stopping power in transmission of $\mathrm{H}$ and $\mathrm{He}$ through thin $\mathrm{LiF}$ films as a function of projectile velocity.

Details on the experimental setups (experiments were performed in two different laboratories) can be found elsewhere [1-3,11]. In brief, ions with atomic numbers $Z_{1}=1-20$ produced in an electron-cyclotronresonance-ion (ECR-ion) source are accelerated to $6 \mathrm{keV} / \mathrm{amu}(v=0.5$ a.u. $)$ and scattered under grazing angle of incidence $\Phi_{\text {in }} \approx 1^{\circ}$ from a $\operatorname{LiF}(001)$ surface at a base pressure of $10^{-10}$ mbar. In order to avoid macroscopic charging-up, the target is kept at a temperature of about $300{ }^{\circ} \mathrm{C}$. The energy loss of specularly reflected ions (i.e., angle of incidence $\Phi_{\text {in }}$ is equal to the angle of exit $\Phi_{\text {out }}$ ) is obtained from energy spectra recorded with an electrostatic analyzer (energy resolution $\left.\delta E / E<10^{-3}\right)$.

The stopping for transmission through self-supporting carbon foils of $(2.80 \pm 0.15) \mu \mathrm{g} / \mathrm{cm}^{2}$ covered by a thin evaporated layer of polycrystalline $\mathrm{LiF}\left(2-4 \mu \mathrm{g} / \mathrm{cm}^{2}\right.$ thick [12]) is measured with a time-of-flight (TOF) setup of about $10^{-3}$ energy resolution. The thickness of the $\mathrm{LiF}$ film was determined by a quartz microbalance monitor and Rutherford backscattering. The energy lost in the insulator is obtained from measurements with covered and clean carbon foils. The effects of multiple scattering (increase of path length and nuclear energy loss) are estimated by using the TRIM code [13] to be smaller than $5 \%$ and corrected accordingly.

Using an averaged surface potential, based on the universal interatomic potential (Ziegler-Biersack-Littmark screening [13]) for describing the surface experiments, we find that trajectories do not significantly depend on the type of projectile for the specific angle of incidence and velocity. This implies that the measured dependence of 
the energy loss on the projectile atomic number $Z_{1}$ can be directly related to a dependence of the stopping power on $Z_{1}$. This allows us to approximate the energy loss $\Delta E$ by

$$
\Delta E=L \frac{d E}{d x},
$$

where $d E / d x$ is the stopping power for the ion and $L$ is the effective interaction length, i.e., the part of the trajectory in which the ion interacts efficiently with the surface.

The electronic stopping power for different $Z_{1}$ ions traveling through an electron gas is obtained from the transport cross section at the Fermi level [14]:

$$
\frac{d E}{d x}=v n_{0} v_{F} \sigma_{\mathrm{tr}}\left(v_{F}\right)=v Q\left(v_{F}\right),
$$

where $v_{F}=\left(3 \pi^{2} n_{0}\right)^{1 / 3}$ is the Fermi velocity. The friction coefficient $Q$ is proportional to the density of electrons $\left(n_{0}\right)$ and to the transport cross section at the Fermi level $\left(\sigma_{\text {tr }}\right)$ of the corresponding screened potential, which is calculated within density-functional theory (DFT) as applied to a static impurity in an electron gas [15]. The energy loss in the low ion velocity limit, given by Eq. (2), is a result of the momentum transfer to a uniform current of electrons scattered by a fixed (impurity) potential. This formalism has been successfully applied to the energy loss of low energy ions in metals [16], where the uniform current of electrons corresponds to the conduction band electrons of the metal in the reference frame of the ion [4]. Even in the case of transition metals, where the $d$ electrons contribute to the energy loss process, the free electron gas model has been shown to explain the measured data when an appropriate value of $n_{0}$ is chosen [16]. This value represents the effective number of electrons contributing to the stopping.

Figure 1 shows the measured energy loss as a function of $Z_{1}$ for specularly reflected ions under $\Phi_{\text {in }}=1^{\circ}$. $Z_{1}$ oscillations similar to those in metals [11] are observed, reflecting the shell structure of the projectile levels: a minimum appears at $Z_{1}=12$ shifted from the noble gas value $\left(Z_{1}=10\right)$ due to screening, and a maximum about $Z_{1}=8-9$ via a $2 p$ resonance. We compare the measured energy loss data with electronic friction coefficients $Q$ for $r_{s}=1.5 . r_{s}=\sqrt[3]{3 /\left(4 \pi n_{0}\right)}$. The agreement between the experimental and theoretical results is fairly good. We deduce from relation (1), and by using the measured data, a value about 85 a.u. of the interaction length $L$ for all of the $Z_{1}$ values.

In Fig. 2 the experimental data for foil transmission are compared with theoretical results obtained within DFT for a free electron gas with $r_{s}=1.5$. Good agreement is found for both $\mathrm{H}$ and $\mathrm{He}$. A linear velocity dependence of the stopping power is clearly observable, which reflects the lack of a noticeable influence of the band gap. In transmission experiments the stopping power is obtained directly from measurements of the energy loss and target thickness.

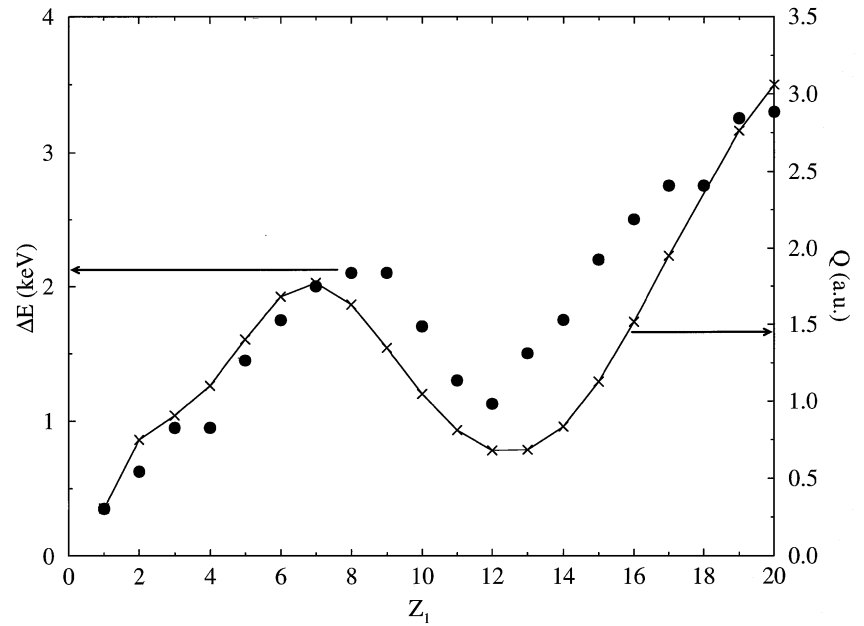

FIG. 1. Energy loss (in keV) of $v=0.5$ a.u. projectiles scattered off a $\mathrm{LiF}(100)$ surface under grazing angle of incidence $\Phi_{\text {in }}=1^{\circ}$ as a function of the projectile atomic number $Z_{1}$ (solid circles). The solid line joins the theoretical results obtained for the friction coefficient $Q$ (in a.u.) with $r_{s}=1.5$. Note that the interaction length $L$ is chosen to 85 a.u. in order to best match the energy loss $\Delta E=L \times v \times Q$.

Note that the same density parameter, $r_{s}=1.5$, reproduces the experimental data obtained in transmission as well as in surface scattering. So, it is tempting to assume that in both cases comparable mechanisms are responsible for projectile energy loss. Moreover, $r_{s}=1.5$ is the value that one obtains when considering the $2 p$ and $2 s$ electrons of $F^{-}$as free electrons. This value is also consistent with the measured bulk plasmon frequency $\left(\omega_{p} \approx 25 \mathrm{eV}\right)$ [17]. We note that the value $r_{s}=1.5$ corresponds to a rather

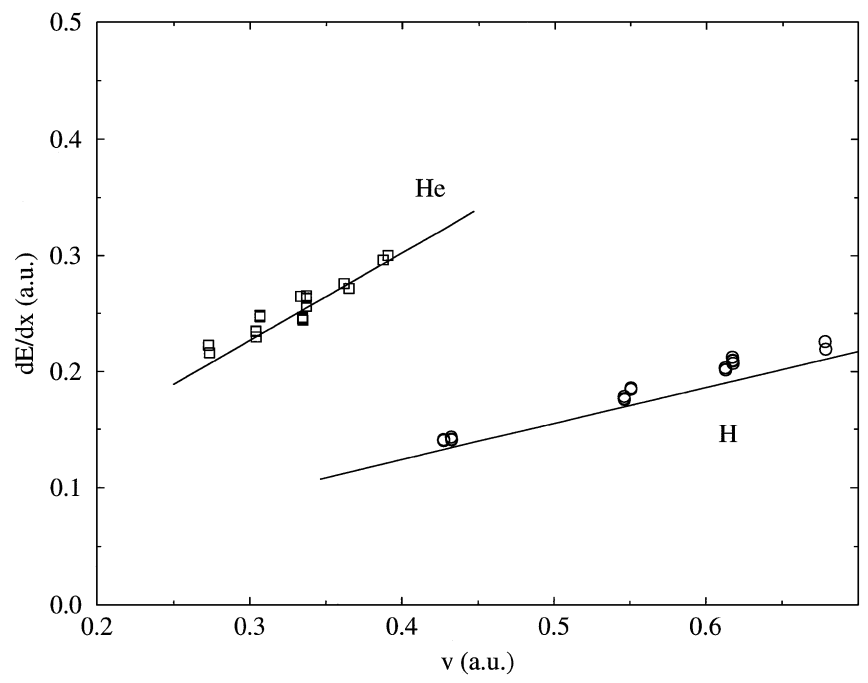

FIG. 2. Stopping power (in a.u.) of $\mathrm{H}$ and He ions traveling through a LiF target as a function of the velocity of the projectiles. The circles correspond to the experimental data obtained in transmission for $\mathrm{H}$ projectiles and the squares to He projectiles. The theoretical results (solid lines) are calculated using $r_{s}=1.5$. 
high electronic density, comparable to the one used to describe the stopping in polycrystalline Au [16] and higher than the electronic density of $\mathrm{Al}\left(r_{s}=2.07\right)$. On the other hand, the $Z_{1}$ oscillations that appear in gaseous targets, which have been related to capture and loss cycles, have smaller amplitudes [18].

Since the valence band of $\mathrm{LiF}$ does not constitute a free electron gas, the good agreement between the experiment and our theoretical approach calls for an explanation. The model used in Ref. [3] to interpret the energy loss of slow protons invoked charge transfer processes between (almost) undisturbed target and projectile electronic levels. The large target-projectile distance resulting from the low value of the component of the proton energy normal to the surface in those experiments justifies the assumption of undisturbed levels. However, in the case of our surface scattering experiment the distances of closest approach are about 1.3 a.u. from the topmost atomic layer. The projectiles get so close to the target that molecular orbitals are formed and one can no longer assume that the target and projectile levels remain undisturbed. Obviously, the same argument is valid for the transmission experiments in which a large overlap of the target and projectile electron clouds takes place. Under these conditions, it is not possible to distinguish between electron-hole pairs or excitons (target excitations) and charge exchange processes (projectile and target excitations) since one deals with a complex target-projectile unified system.

From our combined experimental and theoretical work it is concluded that this complex system shows a metallic character $\left(Z_{1}\right.$ oscillations corresponding to $r_{s}=1.5$, no threshold in transmission experiments). Electron promotion via molecular orbital formation leads to a reduction of energy defects in comparison to the unperturbed energy gap of the crystal [2,9]. Furthermore, the existence of electronic levels bound to the projectile supports the use of the density-functional nonlinear screening model for a positive charge. The description of the induced charge density that we use is adequate because it is mostly made of bound states. Therefore, our model describes the average excitations of the complex system in a proper way, although it does not distinguish between target and projectile excitations. In this case, the Fermi velocity has the same meaning as in transition metals; it corresponds to the effective number of electrons participating in the stopping.

Support for this interpretation comes from experiments with slow negative antiparticles $\left(p^{-}, \mu^{-}\right)$that obviously do not form bound states. For instance, in the case of slow negative muons traveling through the insulator kapton, a threshold effect in stopping has been observed below $v \approx 0.3$ a.u. [19] and is related to the energy gap in the target excitation spectrum. A threshold effect has also been observed for protons in grazing scattering at very low energies (below about 1-2 keV) [3], where collisions proceed with impact parameters and velocities such that molecular promotion to vacuum energies plays a negli- gible role. Here, transitions between promotion curves at larger impact parameters give rise to discrete energy losses owing to charge exchange [3] and excitations of surface excitons [10].

In conclusion, for positive slow ions at energies above $2 \mathrm{keV} / \mathrm{amu}$ the energy loss mechanisms in insulators are similar to those in metals and can be described within the same self-consistent model. The low energy ion represents a strong perturbation of the target material, so that the insulator character of the target is lost and it does not make any sense to distinguish between target and projectile levels (excitations). This distinction can be made only for very slow $(E<2 \mathrm{keV} / \mathrm{amu})$ ions under very grazing incidence and negative antiparticles, and effects related to an energy gap in the excitation spectrum can be observed. For transmission experiments, even at very low energies, we expect electron promotion processes to be possible, due to the inevitable overlap of the electron orbitals of the anion $\left(F^{-}\right)$in the crystal lattice and the projectile ion.

J.I.J., A. A., and P.M.E. acknowledge partial support from the Spanish DGICYT (Project No. PB97-0636), Eusko Jaurlaritza, Iberdrola S. A., Gipuzkoako Foru Aldundia, and Euskal Herriko Unibertsitatea. H.W. acknowledges support from the Deutsche Forschungsgemeinschaft under Contract No. Wi-1336. D. S., K. E., and P.B. acknowledge financial support from the Austrian Science fund (FWF) under Contracts No. P10183-PHY and No. P10740-PHY.

*Also at Donostia International Physics Center, San Sebastiàn, Spain.

[1] P. Bauer, W. Rösler, and P. Mertens, Nucl. Instrum. Methods Phys. Res., Sect. B 69, 46 (1992).

[2] K. Eder, D. Semrad, P. Bauer, R. Golser, P. Maier-Komor, F. Aumayr, M. Peñalba, A. Arnau, J. M. Ugalde, and P. M. Echenique, Phys. Rev. Lett. 79, 4112 (1997).

[3] C. Auth, A. Mertens, H. Winter, and A. Borisov, Phys. Rev. Lett. 81, 4831 (1998).

[4] P. M. Echenique, R. M. Nieminem, J. C. Ashley, and R. H. Ritchie, Phys. Rev. A 33, 897 (1986).

[5] R. Golser and D. Semrad, Phys. Rev. Lett. 66, 1831 (1991).

[6] A. Arnau, P. M. Echenique, and R. H. Ritchie, Nucl. Instrum. Methods Phys. Res., Sect. B 33, 138 (1988).

[7] D. Semrad, Phys. Rev. A 33, 1646 (1986).

[8] Atomic units (a.u.) will be used unless otherwise stated.

[9] P. A. Zeijlmans van Emmichoven, A. Niehaus, P. Stracke, P. Wiegershaus, S. Krischok, V. Kempter, A. Arnau, F. J. García de Abajo, and M. Peñalba, Phys. Rev. B 59, 10950 (1999).

[10] P. Roncin, J. Villete, J. P. Atanas, and H. Khemliche, Phys. Rev. Lett. 83, 864 (1999).

[11] H. Winter, C. Auth, A. Mertens, A. Kirste, and M. J. Steiner, Europhys. Lett. 41, 437 (1998).

[12] P. Maier-Komer, G. Dollinger, and E. Hamman, Nucl. Instrum. Methods Phys. Res., Sect. A 303, 88 (1991). 
[13] J. F. Ziegler, J. P. Biersack, and U. Littmark, The Stopping and Range of Ions in Solids (Pergamon Press, New York, 1985).

[14] N.F. Mott and H. Jones, The Theory and Properties of Metal Alloys (Dover, New York, 1958).

[15] E. Zaremba, L. M. Sander, H. B. Shore, and J.H. Rose, J. Phys. F 7, 1763 (1977).
[16] I. Nagy, A. Arnau, and P. M. Echenique, Phys. Rev. A 40, 987 (1989).

[17] J. R. Fields, P. C. Gibbons, and S. E. Schnatterly, Phys. Rev. Lett. 38, 430 (1977).

[18] J. H. Ormrod, Can. J. Phys. 46, 497 (1968).

[19] H. Daniel, F. J. Hartman, W. Neumann, and W. Schott, Phys. Lett. A 191, 155 (1994). 